\title{
Mini-Review
}

\section{RTX toxins in Pasteurellaceae}

\author{
Joachim Frey, Peter Kuhnert
}

Institute for Veterinary Bacteriology, University of Bern, Switzerland

Received November 19, $2001 \cdot$ Revision received January 14, 2002 · Accepted January 14, 2002

\begin{abstract}
RTX toxins (repeats in the structural toxin) are pore-forming protein toxins produced by a broad range of pathogenic Gram-negative bacteria. In vitro, RTX toxins mostly exhibit a cytotoxic and often also a hemolytic activity. They are particularly widespread in species of the family Pasteurellaceae which cause infectious diseases, most frequently in animals but also in humans. Most RTX toxins are proteins with a molecular mass of $100-200 \mathrm{kDa}$ and are post-translationally activated by acylation via a specific activator protein. The repeated structure of RTX toxins, which gave them their name, is composed of iterative glycine-rich nonapeptides binding $\mathrm{Ca}^{2+}$ on the C-terminal half of the protein. Genetic analysis of RTX toxins of various species of Pasteurellaceae and of a few other Gram-negative bacteria gave evidence of horizontal transfer of genes encoding RTX toxins and led to speculations that RTX toxins might have originated from Pasteurellaceae. The toxic activities of RTX toxins in host cells may lead to necrosis and apoptosis and the underlying detailed mechanisms are currently under investigation. The impact of RTX toxins in pathogenicity and the immune responses of the host were described for several species of Pasteurellaceae. Neutralizing antibodies were shown to significantly reduce the cytotoxic activity of RTX toxins. They constitute a valuable strategy in the development of immuno-prophylactics against several animal diseases caused by pathogenic species of Pasteurellaceae. Although many RTX toxins possess cytotoxic and hemolytic activities toward a broad range of cells and erythrocytes, respectively, a few RTX toxins were shown to have cytotoxic activity only against cells of specific hosts and/or show cell-type specificity. Further evidence exists that RTX toxins play a potential role in host specificity of certain pathogens.
\end{abstract}

Key words: RTX toxins - Pasteurellaceae - pathogenicity - host specificity

\section{Introduction}

Bacterial RTX toxins, named by their repeats in the structural toxin peptide (Strathdee and Lo, 1989), constitute a homogenous family of pore-forming toxins of Gram-negative bacteria. The hemolytic activity is the most obvious phenotype of many RTX toxins under in vitro conditions. However, the erythrocytes might not be the main targets of RTX

Corresponding author: Joachim Frey, Institute for Veterinary Bacteriology, University of Bern, Länggass-Strasse 122 , CH-3012 Bern, Switzerland. Phone: +41316312368, Fax: + 41316312634 , E-mail: joachim.frey@vbi.unibe.ch 


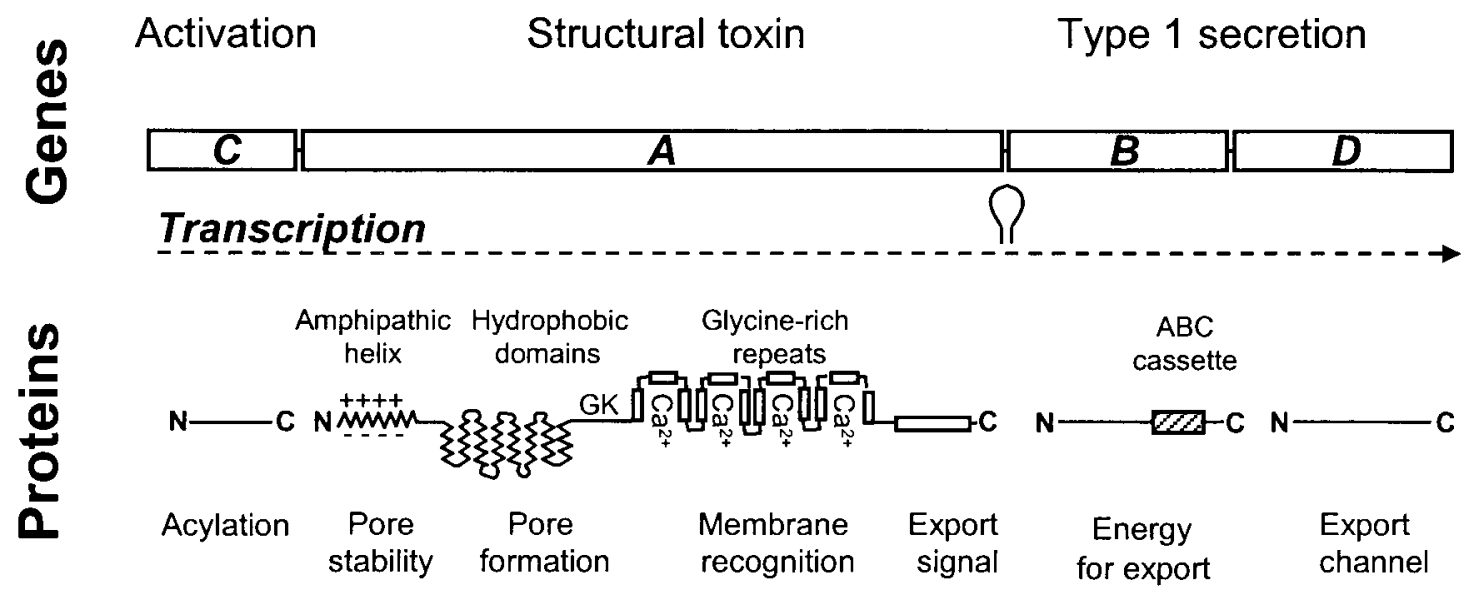

Fig. 1. Genetic organisation and structure of RTX toxins. This figure represents the most common genetic and structural organisation of RTX toxins and is mainly based on that of the E. coliHlyA (Hughes et al., 1992b; Ludwig et al., 1988). The genetic organisation of RTX toxins is shown on the top. The four genes of the operon are represented by open boxes with the activator gene $C$, the gene encoding the structural toxin $A$, and the secretion genes $B$ and $D$. The dashed line below the gene map shows the direction of transcription. The rho-independent transcription termination signal between the $A$ and $B$ gene is indicated by a hairpin. The gene products with their major structural characteristics are represented below the corresponding genes. The domains of the structural toxin protein are annotated. GK indicates the glycine-lysine acylation site(s). The ATP-binding cassette of the secretion protein B is abbreviated by ABC. N indicates the amino-terminal and $\mathrm{C}$ the carboxy-terminal end of the peptides.

toxins. In vivo, RTX toxins induce inflammatory mediators or exert cytotoxic and cytolytic effects mainly to cells of the host's immune defense, thus provoking necrosis, apoptosis, inflammation and disease (Czuprynski and Welch, 1995; Welch, 2001). RTX toxins are postulated by some authors to act in synergy with lipopolysaccharides (Czuprynski and Welch, 1995; Li and Clinkenbeard, 1999) which is currently debated (Welch, 2001). RTX toxins are proteins with a typical molecular mass of $100-$ $200 \mathrm{kDa}$. The pore forming activity was shown for several RTX toxins, but was studied in detail mostly for the Escherichia coli $\alpha$-hemolysin HlyA (Benz et al., 1992; Benz et al., 1994; Ludwig et al., 1991, 1993; Maier et al., 1996). In contrast to the concept of pore forming activity of RTX toxins, one study which was done with the E. coli $\alpha$-hemolysin HlyA indicated that HlyA is inserted in the target membrane as an integral protein, thus occupying only one of the membrane phospolipid monolayers, thus excluding a trans-membrane location which is incompatible with pore forming activity (Soloaga et al., 1999). The consensus structure of most RTX toxins is shown in Figure 1. It shows, at the Nterminal end, a strong membrane-active amphipathic helix structure presumably initiating the contact with the host cell, followed by large hydrophobic domains which were shown to create pores in lipid bi-layers (Ludwig et al., 1991, 1993). The central part of the protein contains one or several acylation sites characterized by glycine-lysine, where the lysine residue is acylated post-translationally by means of a specific activator protein $\mathrm{C}$ encoded on the same operon as the structural toxin $\mathrm{A}$ (Hughes et al., 1992a; Ludwig et al., 1996; Stanley et al., 1998). The repeated structure of RTX toxins, which gave them their name, is constituted by $9-47$ copies of iterative glycine-rich nonapeptides on the C-terminal half of the protein (Fig. 1). These glycinerich repeats have a strong capacity to bind $\mathrm{Ca}^{2+}$, which was shown to be involved in binding to erythrocytes together with the lipidated amino acid residues (Hughes et al., 1992b; Ludwig et al., 1988). The 30-50 most C-terminal amino acids of the structural toxin protein constitute the signal for the specific type I secretion system which is encoded by two genes, $B$ and $D$, both located on the same operon as the activator gene $C$, and the structural toxin gene $A$, organized in the manner CABD (Hughes et al., 1992b) (Fig. 1). Probably due to its strong hemolytic activity and the ease to genetically manipulate Escherichia coli, the $\alpha$-hemolytic HlyA of E. coli is the RTX toxin first discovered and best studied. In particular, the E. coli HlyA was used as a model to study the stoichiometry of the transmembrane pore and its mechanism of formation (Ludwig et al., 1993), and the mechanisms of ATPase-driven type I secretion of HlyB and HlyD in interaction with the host bacterium outer membrane protein TolC (Andersen et al., 2000; Balakrishnan et al., 2001; Thanabalu et al., 1998). A well detailed comprehensive and critical review on basic molecular aspects of 
structure and function of RTX toxins and their in vivo function in cell killing has recently been published (Welch, 2001).

RTX toxins are particularly widespread among species of Pasteurellaceae. This family of bacteria comprises evolutionary closely related bacteria which are found mainly in animals and also in man, either as commensals or as pathogens causing similar types of infectious diseases affecting mucosal tissue (Donachie et al., 1995). The current review intends to give an overview on the occurrence and the spread of RTX toxins in species of Pasteurellaceae, to summarize their phenotypes and to discuss their potential role in pathogenesis and host specificity.

\section{Phylogeny, spread and in vitro phenotypes of RTX toxins in Pasteurellaceae}

Based on their genetic relationship, as calculated from similarity analysis of the genes encoding the structural toxin A, RTX toxins of Pasteurellaceae can be grouped into four clusters (Fig. 2). These clusters also reflect some of the main in vitro phenotypes of these toxins, including hemolysis and co-hemolysis (CAMP reaction) as well as cytotoxic activity (Table 1 ).

The first cluster of RTX toxin genes is formed by ApxIA of A. pleuropneumoniae (Frey et al., 1993), a pig pathogen causing pleuropneumonia, the toxin LtxA (also named AaltA) of Actinobacillus actinomycetemcomitans causing juvenile periodontitis in human (Lally et al., 1989a) and the hemolysin HlyA of Escherichia coli which is found in human uropathogenic E. coli isolates as well as in pig isolates from various diseases in particular oedema disease (Fig. 2). All these RTX toxins are reported to possess strong cytotoxic activity. ApxIA and HlyA are also strongly hemolytic on medium containing sheep erythrocytes, while LtxA does not show hemolysis on this medium. Although the structural genes of these three RTX toxins show strong similarities of their nucleotide sequences, the bacterial species harboring these three related RTX toxins are phylogenetically distant from each other. Escherichia coli, belonging to the Enterobacteriaceae, is not even a member of the Pasteurellaceae (Fig. 2). This indicates that a horizontal transfer of RTX toxin genes has occurred not only among different species of Pasteurellaceae but also across bacterial families.
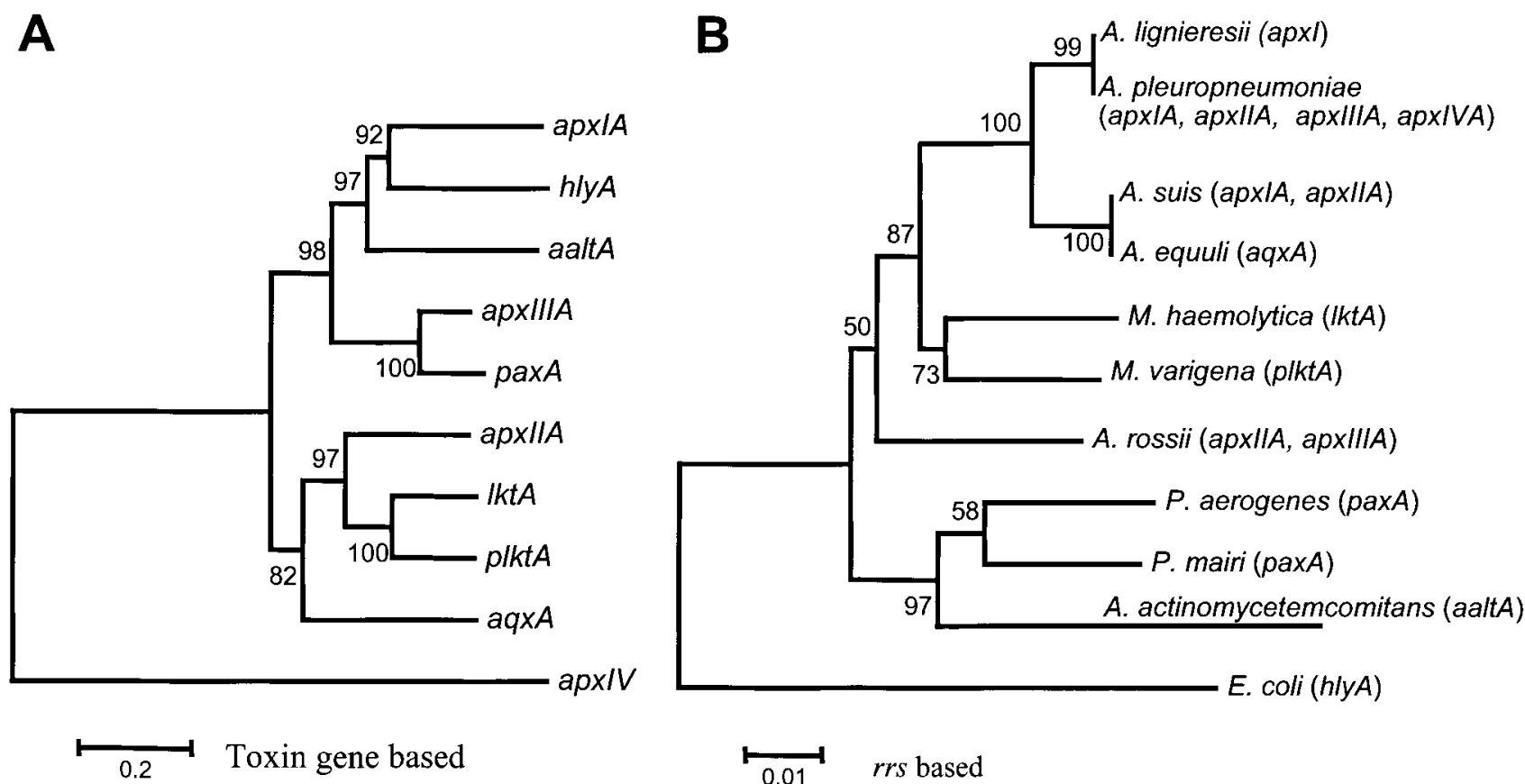

Fig. 2. A. Phylogenetic relationship of structural RTX toxin genes from Pasteurellaceae and E. coli. B. Phylogenetic position of the bacterial species harbouring the corresponding RTX toxin genes (indicated in brackets), based on the 16S rRNA gene (rrs) sequence of the type strains. A distance matrix was calculated by the Jukes-Cantor algorithm (Jukes and Cantor, 1969) and a tree was built by the neighbour-joining method (Saitou and Nei, 1987). Bootstrap values of 500 simulations are given at the branching points of the tree. Scale bars indicate genetic distances. 
Table 1. Characteristics of selected RTX toxins.

\begin{tabular}{|c|c|c|c|c|c|c|}
\hline Toxin & mol. mass & genetic locus & host species & $\begin{array}{l}\text { current phenotypic } \\
\text { characterization }\end{array}$ & $\begin{array}{l}\text { toxin specificity/ } \\
\text { pathogen } \\
\text { specificity }\end{array}$ & Reference \\
\hline HlyA & $107 \mathrm{kDa}$ & hlyCABD & E. coli & $\begin{array}{l}\text { strong haemolytic } \\
\text { strong cytotoxic } \\
\text { pores in lipid bilayers }\end{array}$ & nd*/human & (Felmlee et al., 1985) \\
\hline ApxIA & $110 \mathrm{kDa}$ & $a p x / C A B D$ & $\begin{array}{l}\text { A. pleuropneumoniae } \\
\text { A. suis } \\
\text { A. lignieresii }\end{array}$ & $\begin{array}{l}\text { strong haemolytic } \\
\text { strong cytotoxic } \\
\text { CAMP } \\
\text { pores in lipid bilayers }\end{array}$ & nd/pig & (Frey et al., 1994a) \\
\hline LtxA (AaltA) & $108 \mathrm{kDa}$ & $\begin{array}{l}\text { ltx } C A B D \\
(\text { altCABD) }\end{array}$ & $\begin{array}{l}\text { A. actinomycetem- } \\
\text { comitans }\end{array}$ & leukotoxic & $\begin{array}{l}\text { human, higher } \\
\text { primates/human }\end{array}$ & (Kraig et al., 1990) \\
\hline ApxIIIA & $113 \mathrm{kDa}$ & apxII/CABD & $\begin{array}{l}\text { A. pleuropneumoniae } \\
\text { A. rossii }\end{array}$ & $\begin{array}{l}\text { cytotoxic } \\
\text { macrophage toxic } \\
\text { pores in lipid bilayers } \\
\text { CAMP }\end{array}$ & nd/pig & (Chang et al., 1993b) \\
\hline PaxA & $108 \mathrm{kDa}$ & $\operatorname{pax} C A B D$ & $\begin{array}{l}\text { P. aerogenes } \\
\text { P. mairi }\end{array}$ & CAMP & nd/pig & (Kuhnert et al., 2000) \\
\hline ApxIIA & 110 kDa & apxI/CA & $\begin{array}{l}\text { A. pleuropneumoniae } \\
\text { A. suis } \\
\text { A. rossii } \\
\text { Actinobacillus sp. }\end{array}$ & $\begin{array}{l}\text { weak hemolytic } \\
\text { weak cytotoxic } \\
\text { pores in lipid bilayers } \\
\text { CAMP }\end{array}$ & nd/pig & (Chang et al., 1989) \\
\hline LktA & 102 kDa & IktCABD & M. haemolytica & $\begin{array}{l}\text { hemolytic } \\
\text { leukotoxic }\end{array}$ & cattle/cattle & (Lo et al., 1987) \\
\hline PlktA & 105 kDa & plktCABD & M. varigena & $\begin{array}{l}\text { hemolytic } \\
\text { leukotoxic }\end{array}$ & nd/pig & (Chang et al., 1993a) \\
\hline AqxA & 110 kDa & $\operatorname{aqx} C A B D$ & $\begin{array}{l}\text { A. equuli } \\
\text { A. equuli-like }\end{array}$ & $\begin{array}{l}\text { hemolytic } \\
\text { CAMP }\end{array}$ & nd/horse & (Berthoud et al., 2002) \\
\hline ApxIVA & $170-202 \mathrm{kDa}$ & apxIVA & A. pleuropneumoniae & CAMP & nd/pig & (Schaller et al., 1999) \\
\hline
\end{tabular}

* nd, not defined

The second cluster of RTX toxins of Pasteurellaceae currently includes only two toxins, ApxIIIA of A. pleuropneumoniae (Frey et al., 1993; Rycroft et al., 1991) and PaxA of Pasteurella aerogenes (Kuhnert et al., 2000). These two toxins are nonhemolytic, but they both show a significant CAMP reaction, a co-hemolytic reaction specified by the synergy of certain pore-forming toxins and the sphingomyelinase from $\beta$-hemolytic Staphylococcus aureus (Frey et al., 1994b; Kuhnert et al., 2000). ApxIIIA was shown to be highly cytotoxic against macrophages and was therefore previously also named macrophage toxin (Lally et al., 1989b; Macdonald and Rycroft, 1992; Rycroft et al., 1991). The cytotoxic activity of PaxA has not yet been analyzed. The bacterial species harboring these two very similar toxins are phylogenetically distant species of Pasteurellaceae, which is a further indication of a horizontal transfer of RTX toxin genes in the past.

The third cluster contains the most prominent representative of RTX toxins of Pasteurellaceae, the leukotoxin LktA (Lo et al., 1987) of Mannheimia (Pasteurella) haemolytica, as well as the leukotoxin PlktA of Mannheimia varigena, the ApxIIA toxin of
A. pleuropneumoniae and AqxA of Actinobacillus equuli. LktA plays a role in the pathogenesis of bovine and ovine pneumonic pasteurellosis caused by M. haemolytica (Frank, 1989; Gilmour and Gilmour, 1989). Ten different alleles of the $l k t A$ gene are currently known. They are derived from $M$. haemolytica, Mannheimia glucosida and Pasteurella trehalosi. These alleles have a mosaic structure reflecting horizontal gene transfer and extensive intragenic recombination which is thought to have contributed to host switching and host specificity of the different subtypes of $M$. haemolytica from bovine and ovine hosts (Davies et al., 2001). The leukotoxin PlktA of Mannheimia varigena, a porcine pathogen previously designated as Pasteurella haemolytica-like organism (Chang et al., 1993a), and the ApxIIA toxin of Actinobacillus pleuropneumoniae (Frey et al., 1993), the etiological agent of porcine pleuropneumonia, are genetically close relatives to LktA. ApxIIA is also found in Actinobacillus suis and Actinobacillus rossii, in variant forms (Burrows and Lo, 1992; Schaller et al., 2000). The recently discovered RTX toxin AqxA of Actinobacillus equuli and Actinobacillus equuli-like bacteria from horses (P. Kuhnert, unpublished results) be- 
longs to the same phylogenetic cluster (Fig. 2). All RTX toxins of this cluster show moderate hemolytic activity under in vitro conditions, and most of them were confirmed to possess cytotoxic activity towards cell cultures.

The toxin ApxIV of A. pleuropneumoniae (Schaller et al., 1999) is genetically very distant from any of the other known RTX toxins (Fig. 2). It is not expressed by $A$. pleuropneumoniae under culture conditions, but is produced during infection of pigs with A. pleuropneumoniae. ApxIVA can be expressed as recombinant protein in E. coli, where it shows a slight hemolytic activity and a distinct cohemolytic CAMP reaction. The genetic organization of apxIV also shows strong differences to the other RTX toxins. It lacks the activator gene $C$ and type I secretion genes $B$ and $D$. However, upstream $a p x I V A$ an ORF was discovered, which is necessary for the hemolytic and CAMP activity of the ApxIVA toxin (Schaller et al., 1999). ApxIVA, which is specific to the species A. pleuropneumoniae (Schaller et al., 2001), shows high similarity to the RTX protein FrpC of Neisseria meningitidis, a protein that induces high levels of serum antibodies during invasive disease (Osicka et al., 2001).

\section{Role of RTX toxins in pathogenicity}

The role of RTX toxins in the pathogenicity of pasteurellosis has been studied for a few species either indirectly by molecular epidemiological investigations, by in vitro experiments of purified toxins on cultured host cells, or directly by experimental infection of animals with isogenic strains where the corresponding toxin gene was deleted. The leukotoxin LktA of M. haemolytica was investigated intensively. Purified LktA was shown to induce the release of histamine from bovine pulmonary mast cells (Adusu et al., 1994), to cause cell swelling and rapid leakage of $\mathrm{K}^{+}$from isolated bovine neutrophils (Clinkenbeard et al., 1989) and to cause increased synthesis of leukotriene B-4, accompanied by neutrophil lysis (Clinkenbeard et al., 1994). Makeswaran et al. (1992) observed that culture supernatants of M. haemolytica, which contained LktA, stimulated an immediate neutrophil respiratory burst, as measured by the generation of oxygen-derived free radicals. LktA was demonstrated to bind to beta-2 integrins on sensitive bovine leukocytes, very likely via CD18 (Ambagala et al., 1999) (Jeyaseelan et al., 2000; Leite et al., 2000), and it very rapidly leads to processes of apoptosis, including DNA fragmentation (Stevens and Czu- prynski, 1996; Sun et al., 1999). Deletion mutants $(\Delta l k t A)$ of $M$. haemolytica revealed reduction in virulence compared to their isogenic wild-type strains, but were not avirulent, as measured by clinical symptoms and lung lesion scores in an experimentally infected calf model of pneumonic pasteurellosis (Tatum et al., 1998). Consequently, LktA is involved in pathogenicity of M. haemolytica, but it does not seem to be essential for the cause of the disease.

The leukotoxin LtxA (AaltA) of A. actinomycetemcomitans was also demonstrated to bind beta-2 integrin of human target cells (Lally et al., 1997) and to be able to kill human HL-60 by inducing apoptosis (Korostoff et al., 1998). It is believed that the elimination of acute inflammatory cells by LtxA plays a critical role in the pathogenesis of periodontitis caused by A. actinomycetemcomitans. Differences in the promoters of $l t x A$ genes, due to the presence or the absence of a 530-bp segment in different strains, are responsible for low, respectively high expression strains of LtxA (Brogan et al., 1994). However, this significant difference of LtxA production does not seem to be related to the severeness of periodontitis in the patient from whom the strains were isolated (Contreras et al., 2000), and hence questions the impact of this toxin in pathogenesis.

The role of the different Apx toxins of $A$. pleuropneumoniae in the pathogenesis of porcine pleuropneumonia was studied with deletion mutants in the apx toxin genes and in trans-complementation experiments. Using the highly virulent $A$. pleuropneumoniae serotype 1 type strain 4074, which produces and secretes ApxIA and ApxIIA, Jansen and collaborators showed that the parent strain or those mutants that secreted either ApxIA or ApxIIA provoked an oxidative burst and killed porcine neutrophils, whereas double mutants $(\triangle a p x I A+\Delta a p x I I A)$ did not (Jansen et al., 1995). Direct evidence for the role of ApxIA and ApxIIA in the pathogenesis of porcine pleuropneumonia was obtained from experimental infection of pigs with either a virulent strain JM5 of A. pleuropneumoniae serotype 5 or several derivatives of the same. The wild-type strain JM5 secreted ApxIA and ApxIIA and was fully virulent. A deletion mutant, which was unable to secrete ApxIA and ApxIIA, was nonpathogenic for experimentally infected pigs. Reintroduction of the genes allowing secretion of ApxIIA resulted in a strain of moderate virulence, while complementation with all genes for biosynthesis and secretion of ApxIA and ApxIIA restored full virulence. This showed that ApxIIA is essential in the pathogenesis of porcine pleuropneumonia and 
that the combination of secreted ApxIA and ApxIIA by certain serotypes of A. pleuropneumoniae is responsible for the very severe symptoms and the mostly fatal course of the disease (Reimer et al., 1995). These results were confirmed by intratracheal infusion of purified toxins ApxIA and ApxIIA to pigs (Kamp et al., 1997). Antibodies directed against ApxIA, ApxIIA and ApxIIIA were shown to neutralize the hemolytic and cytotoxic activities of these toxins (Udeze and Kadis, 1992) and to protect mice against experimental infection with A. pleuropneumoniae (Bhatia et al., 1991). Finally, inactivated ApxIA, ApxIIA and ApxIIIA were shown to confer protective immunity against $A$. pleuropneumoniae to pigs and were used as antigens in potent vaccines (Kobisch and van den Bosch, 1992; van den Bosch et al., 1990). Although ApxIA and ApxIIA were shown to be essential virulence factors of $A$. pleuropneumoniae, it should be noted that Actinobacillus suis and Actinobacillus rossii, both species of low pathogenicity that are occasionally isolated from pigs, particularly from early weaned piglets, also secrete ApxIA and ApxIIA or ApxIIA and ApxIIIA, respectively (Burrows and Lo, 1992; Schaller et al., 2000). Consequently, ApxIA, ApxIIA and ApxIIIA do not seem to be sufficient for pathogenicity of A. pleuropneumonie. It has to be determined whether the A. pleuropneumoniae-specific ApxIV toxin might be the crucial factor that distinguishes this species as a significant pathogen from the various related Actinobacillus species that are not primary pathogens.

The role in pathogenicity of the RTX toxin PaxA, which is produced by certain strains of Pasteurella aerogenes and Pasteurella mairi, has not yet been investigated in detail. However, $P$. aerogenes and $P$. mairi, which were isolated from abortion or neonatal septicemia of pigs, always produced PaxA. Contrary to this, strains from other samples, where these species were found as opportunistic pathogens or as commensals, were devoid of the paxA gene (Kuhnert et al., 2000) (P. Kuhnert, unpublished results), thus indicating that PaxA would be necessary for $P$. aerogenes and $P$. mairi to cause systemic infections.

The role of the recently discovered AqxA in the virulence of the equine pathogen $A$. equuli and related strains remains to be elucidated. This RTX toxin is only present in certain A. equuli and A. equuli-like isolates from horses and is not found in others, indicating that it might contribute to infectivity or persistence in the host. Nevertheless, there is no clear evidence for a correlation of the presence of AqxA and the pathogenicity of $A$. equuli until now. Moreover, hemolytic A. equuli, also referred to as
Bisgaard Taxon 11, is reported to be commonly detected as part of the bacterial flora of horses (Bisgaard et al., 1984).

\section{Host specificity}

The most apparent phenotype of RTX toxins is the hemolytic or co-hemolytic (CAMP) activity manifested by cultivating the corresponding bacteria on standard nutrient blood agar which generally contains sheep erythrocytes. Consequently, the hemolytic activity of RTX toxins shows no species specificity. The cytotoxic activity of some RTX toxins, however, was shown to be specific for cells of the host species of the corresponding pathogen. Thus LtxA of the human pathogen A. actinomycetemcomitans kills specifically human and higher primate monomyelocytes (Lally et al., 1989a), while LktA of M. haemolytica, which causes bovine disease, kills bovine lymphoid cells (Forestier and Welch, 1991; Sun et al., 1999). Using recombinant chimeric ltxA/ $l k t A$ toxin genes allowed to determine the critical area of the A. actinomycetemcomitans LtxA toxin for recognition of human target cells which was located in the segment containing the glycine-rich nonapeptide repeats (Lally et al., 1994).

The E. coli hemolysin HlyA was shown to be cytolytic to sheep erythrocytes, BL3 bovine leukemia-derived B-lymphocytes and human B-cell lymphoma cells Raji. In contrast, the M. haemolytica LktA leukotoxin only lyses bovine BL3 cells of the three above-named cell types. Hybrid constructs between the $h l y A$ and the $l k t A$ genes allowed to determine the domain of HlyA responsible for the hemolytic activity and the domain of LktA that determines the specificity for ruminant cells (Forestier and Welch, 1991). Most recent studies on the RTX toxin ligand-LFA-1 receptor interactions of LtxA and LktA led to speculations that sequence divergence among RTX toxins and the CD11a and CD18 subunits as receptors might account for the different host specificities observed for certain RTX toxins (Jeyaseelan et al., 2000; Lally et al., 1997; Welch, 2001).

Although there are no molecular data on the host specificity of most of the RTX toxins from animal pathogens except for LktA, epidemiological considerations indicate host specificity for other RTX toxins, too. In this respect it is interesting to compare the pig pathogen $A$. suis and the horse-specific $A$. equuli which is associated to sleepy foal disease. Both $A$. suis and A. equuli are phylogenetically very closely related Pasteurellaceae, based on sequence 
analyses of their identical $r r s$ (16S rRNA) genes and other housekeeping genes (Dewhirst et al., 1992) (P. Kuhnert, unpublished results). Analysis of a large number of $A$. suis isolates from pigs revealed specifically the presence of $a p x I C A B D_{\text {var.suis }}$ and $a p x I I C A_{\text {var.suis }}$ genes and correspondingly the production of ApxIA and ApxIIA (VanOstaaijen et al., 1997). In contrast, hemolytic $A$. equuli and $A$. equuli-like bacteria isolated from horses specifically contained the aqxCABD operon of the newly discovered AqxA toxin (P. Kuhnert, unpublished results). This indicates that ApxIA and ApxIIA contribute to the host specificity for pigs of A. suis, A. pleuropneumoniae and A. rossii, which belong to different phylogenetic subgroups of Actinobacillus. In contrast, AqxA seems to direct the host specificity of $A$. equuli and A. equuli-like bacteria in horses. It has to be noted that hemolytic $A$. equuli were often erroneously referred to as equine $A$. suis based on their hemolytic phenotype which is not due to the same RTX toxin (Bisgaard et al., 1984; Samitz and Biberstein, 1991). The speculations which are derived from molecular epidemiological observations, however, must be confirmed further by in vitro experiments with specific equine and porcine cells or in vivo experiments. For these purposes swapping of corresponding RTX genes from A. suis and A. equuli and testing in animal models the susceptibility of the host for the corresponding wild-type or toxinrecombinant strain could be considered.

\section{Concluding remarks}

RTX toxins are found among a broad diversity of Pasteurellaceae, mainly of the genus Actinobacillus, Mannheimia and Pasteurella. Most RTX toxin genes exhibit a GC:AT ratio which ranges between $36 \%$ and $46 \%$ GC and which is close to that of the genomes of Pasteurellaceae (around 40\% GC). This indicates that RTX toxin genes might originate from ancestral Pasteurellaceae species from where they spread also to other bacterial families during evolution (see also (Welch, 1991)).

Although RTX toxins might act as virulence factors of several pathogens, they seem in most cases not to be essential for pathogenicity of these species. While they might contribute significantly to the severeness of the diseases caused by their host bacteria, in many cases they appear to act together with other virulence determinants. Moreover, the occurrence of specific RTX toxins in species of low pathogenicity or even avirulent species shows that the virulence potential of a bacterium is not given by the presence of an RTX toxin alone.

Currently, the most detailed results on the molecular mechanisms have been obtained on RTX toxins the impact of which in pathogenicity of their respective host bacterium is not very strong. It seems therefore worthwhile to consider also more detailed molecular studies of those RTX toxins which seem to play a crucial role in the virulence of their host pathogens. Furthermore, it is worthwhile to discuss in the future an additional biological role of RTX toxins, which is their putative potential to drive or determine host-specificity of the pathogen.

\section{References}

Adusu, T. E., Conlon, P. D., Shewen, P. E., Black, W. D.: Pasteurella haemolytica leukotoxin induces histamine release from bovine pulmonary mast cells. Can. J. Vet. Res. 58, 1-5 (1994).

Ambagala, T. C., Ambagala, A. P., Srikumaran, S.: The leukotoxin of Pasteurella haemolytica binds to beta(2) integrins on bovine leukocytes. FEMS Microbiol. Lett. 179, $161-167$ (1999).

Andersen, C., Hughes, C., Koronakis, V.: Chunnel vision. Export and efflux through bacterial channeltunnels. EMBO Rep. 1, 313-318 (2000).

Balakrishnan, L., Hughes, C., Koronakis, V.: Substratetriggered recruitment of the TolC channel-tunnel during type I export of hemolysin by Escherichia coli. J. Mol. Biol. 313, 501-510 (2001).

Benz, R., Dobereiner, A., Ludwig, A., Goebel, W.: Haemolysin of Escherichia coli - comparison of pore-forming properties between chromosome and plasmid-encoded haemolysins. FEMS Microbiol. Immunol. 105, 55-62 (1992).

Benz, R., Maier, E., Ladant, D., Ullmann, A., Sebo, P.: Adenylate cyclase toxin (CyaA) of Bordetella pertussis - Evidence for the formation of small ion-permeable channels and comparison with HlyA of Escherichia coli. J. Biol. Chem. 269, 27231 -27239 (1994).

Berthoud, H., Frey, J., Kuhnert, P: Characterization of Aqx and its operon: the hemolytic RTX determinant of Actinobacillus equuli. Vet. Microbiol. 87, 159174 (2002).

Bhatia, B., Mittal, K. R., Frey, J.: Factors involved in immunity against Actinobacillus pleuropneumoniae in mice. Vet. Microbiol. 29, 147-158 (1991).

Bisgaard, M., Piechulla, K., Ying, Y. T., Frederiksen, W., Mannheim, W.: Prevalence of organisms described as Actinobacillus suis or haemolytic Actinobacillus equuli in the oral cavity of horses. Comparative investigations of strains obtained and porcine strains of A. suis sensu stricto. Acta Pathol. Microbiol. Immunol. Scand. 92, 291-298 (1984).

Brogan, J. M., Lally, E. T., Poulsen, K., Kilian, M., Demuth, D. R.: Regulation of Actinobacillus actino- 
mycetemcomitans leukotoxin expression: analysis of the promoter regions of leukotoxic and minimally leukotoxic strains. Infect. Immun. 62, 501 - 508 (1994).

Burrows, L. L., Lo, R. Y.: Molecular characterization of an RTX toxin determinant from Actinobacillus suis. Infect. Immun. 60, 2166-2173 (1992).

Chang, Y. F., Ma, D. P., Shi, J., Chengappa, M. M.: Molecular characterization of a leukotoxin gene from a Pasteurella haemolytica-like organism, encoding a new member of the RTX toxin family. Infect. Immun. 61, 2089-2095 (1993a).

Chang, Y. F., Shi, J. R., Ma, D. P., Shin, S. J., Lein, D. H.: Molecular analysis of the Actinobacillus pleuropneumoniae RTX toxin-III gene cluster. DNA Cell Biol. 12, 351-362 (1993b).

Chang, Y. F., Young, R., Struck, D. K.: Cloning and characterization of a hemolysin gene from Actinobacillus (Haemophilus) pleuropneumoniae. DNA 8, 635-647 (1989).

Clinkenbeard, K. D., Clarke, C. R., Hague, C. M., Clinkenbeard, P., Srikumaran, S., Morton, R. J.: Pasteurella haemolytica leukotoxin-induced synthesis of eicosanoids by bovine neutrophils in vitro. J. Leukocyte Biol. 56, 644-649 (1994).

Clinkenbeard, K. D., Mosier, D. A., Confer, A. W.: Effects of Pasteurella haemolytica leukotoxin on isolated bovine neutrophils. Toxicon 27, 797-804 (1989).

Contreras, A., Rusitanonta, T., Chen, C., Wagner, W. G., Michalowicz, B. S., Slots, J.: Frequency of 530-bp deletion in Actinobacillus actinomycetemcomitans leukotoxin promoter region. Oral Microbiol. Immunol. 15, 338-340 (2000).

Czuprynski, C. J., Welch, R. A.: Biological effects of RTX toxins: The possible role of lipopolysaccharide. Trends Microbiol. 3, 480-483 (1995).

Davies, R. L., Whittam, T. S., Selander, R. K.: Sequence diversity and molecular evolution of the leukotoxin $(l k t A)$ gene in bovine and ovine strains of Mannheimia (Pasteurella) haemolytica. J. Bacteriol. 183, 1394-1404 (2001).

Dewhirst, F. E., Paster, B. J., Olsen, I., Fraser, G. J.: Phylogeny of 54 representative strains of species in the family Pasteurellaceae as determined by comparison of $16 \mathrm{~S}$ rRNA sequences. J. Bacteriol. 174, $2002-$ 2013 (1992).

Donachie, W., Lainson, F. A., Hodgson, J. C. (eds.): Haemophilus, Actinobacillus, and Pasteurella. Plenum Press, New York 1995.

Felmlee, T., Pellett, S., Welch, R. A.: Nucleotide sequence of an Escherichia coli chromosomal hemolysin. J. Bacteriol. 163, 94-105 (1985).

Forestier, C., Welch, R. A.: Identification of RTX toxin target cell specificity domains by use of hybrid genes. Infect. Immun. 59, $4212-4220$ (1991).

Frank, G. H.: Pasteurellosis of cattle. In: Pasteurella and pasteurellosis (C. F. Adlam, J. M. Rutter, eds.), pp. 197-222. Academic Press, London 1989.

Frey, J., Bosse, J. T., Chang, Y. F., Cullen, J. M., Fenwick, B., Gerlach, G. F., Gygi, D., Haesebrouck,
F., Inzana, T. J., Jansen, R., Kamp, E. M., Macdonald, J., MacInnes, J. I., Mittal, K. R., Nicolet, J., Rycroft, A. N., Segers, R. P. A. M., Smits, M. A., Stenbaek, E., Struck, D. K., van den Bosch, J. F., Willson, P. J., Young, R.: Actinobacillus pleuropneumoniae RTX-toxins - Uniform designation of haemolysins, cytolysins, pleurotoxin and their genes. J. Gen. Microbiol. 139, 1723-1728 (1993).

Frey, J., Haldimann, A., Nicolet, J., Boffini, A., Prentki, P.: Sequence analysis and transcription of the apxI operon (hemolysin I) from Actinobacillus pleuropneumoniae. Gene 142, 97-102 (1994a).

Frey, J., Kuhn, R., Nicolet, J.: Association of the CAMP phenomenon in Actinobacillus pleuropneumoniae with the RTX toxins ApxI, ApxII and ApxIII. FEMS Microbiol. Lett. 124, 245-251 (1994b).

Gilmour, N. J. L., Gilmour, J. S.: Pasteurellosis of sheep. In: Pasteurella and pasteurellosis (C. F. Adlam, J. M. Rutter, eds.), pp. 223-261. Academic Press, London 1989.

Hughes, C., Issartel, J. P., Hardie, K., Stanley, P., Koronakis, E., Koronakis, V.: Activation of Escherichia coli prohemolysin to the membrane-targetted toxin by HlyC-directed ACP-dependent fatty acylation. FEMS Microbiol. Immunol. 105, 37-44 (1992a).

Hughes, C., Stanley, P., Koronakis, V.: E. coli hemolysin interactions with prokaryotic and eukaryotic cell membranes. Bioessays 14, 519-525 (1992b).

Jansen, R., Briaire, J., Smith, H. E., Dom, P., Haesebrouck, F., Kamp, E. M., Gielkens, A. L. J., Smits, M. A.: Knockout mutants of Actinobacillus pleuropneumoniae serotype 1 that are devoid of RTX toxins do not activate or kill porcine neutrophils. Infect. Immun. 63, 27-37 (1995).

Jeyaseelan, S., Hsuan, S. L., Kannan, M. S., Walcheck, B., Wang, J. F., Kehrli, M. E., Lally, E. T., Sieck, G. C., Maheswaran, S. K.: Lymphocyte function-associated antigen 1 is a receptor for Pasteurella haemolytica leukotoxin in bovine leukocytes. Infect. Immun. 68, $72-79$ (2000).

Jukes, T. H., Cantor, C. R.: Evolution of protein molecules. In: Mammalian protein metabolism (H. N. Munro, ed.), Vol. 3, pp. 21-132. Academic Press AP, New York 1969.

Kamp, E. M., Stockhofe Zurwieden, N., van Leengoed, L. G., Smits, M. A.: Endobronchial inoculation with Apx toxins of Actinobacillus pleuropneumoniae leads to pleuropneumonia in pigs. Infect. Immun. 65, 4350 - 4354 (1997).

Kobisch, M., van den Bosch, J. F.: Efficacy of an Actinobacillus pleuropneumoniae subunit vaccine. Proceedings of the International Pig Veterinary Society Congress 12, 216 (1992).

Korostoff, J., Wang, J. F., Kieba, I., Miller, M., Shenker, B. J., Lally, E. T.: Actinobacillus actinomycetemcomitans leukotoxin induces apoptosis in HL-60 cells. Infect. Immun. 66, 4474-4483 (1998).

Kraig, E., Dailey, T., Kolodrubetz, D.: Nucleotide sequence of the leukotoxin gene from Actinobacillus 
actinomycetemcomitans: homology to the alpha-hemolysin/leukotoxin gene family. Infect. Immun. 58, 920-929 (1990).

Kuhnert, P., Heyberger-Meyer, B., Nicolet, J., Frey, J.: Characterization of PaxA and its operon: a cohemolytic RTX toxin determinant from pathogenic Pasteurella aerogenes. Infect. Immun. 68, 6-12 (2000).

Lally, E. T., Golub, E. E., Kieba, I. R.: Identification and immunological characterization of the domain of Actinobacillus actinomycetemcomitans leukotoxin that determines its specificity for human target cells. J. Biol. Chem. 269, 31289 -31295 (1994).

Lally, E. T., Golub, E. E., Kieba, I. R., Taichman, N. S., Rosenbloom, J., Rosenbloom, J. C., Gibson, C. W., Demuth, D. R.: Analysis of the Actinobacillus actinomycetemcomitans leukotoxin gene. Delineation of unique features and comparison to homologous toxins. J. Biol. Chem. 264, 15451 - 15456 (1989a).

Lally, E. T., Kieba, I. R., Demuth, D. R., Rosenbloom, J., Golub, E. E., Taichman, N. S., Gibson, C. W.: Identification and expression of the Actinobacillus actinomycetemcomitans leukotoxin gene. Biochem. Biophys. Res. Commun. 159, 256-262 (1989b).

Lally, E. T., Kieba, I. R., Sato, A., Green, C. L., Rosenbloom, J., Korostoff, J., Wang, J. F., Shenker, B. J., Ortlepp, S., Robinson, M. K., Billings, P. C.: RTX toxins recognize a beta 2 integrin on the surface of human target cells. J. Biol. Chem. 272, 30463-30469 (1997).

Leite, F., Brown, J. F., Sylte, M. J., Briggs, R. E., Czuprynski, C. J.: Recombinant bovine interleukin1beta amplifies the effects of partially purified Pasteurella haemolytica leukotoxin on bovine neutrophils in a beta(2)-integrin-dependent manner. Infect. Immun. 68, $5581-5586$ (2000).

Li, J., Clinkenbeard, K. D.: Lipopolysaccharide complexes with Pasteurella haemolytica leukotoxin. Infect. Immun. 67, 2920-2927 (1999).

Lo, R. Y., Strathdee, C. A., Shewen, P. E.: Nucleotide sequence of the leukotoxin genes of Pasteurella haemolytica A1. Infect. Immun. 55, 1987-1996 (1987).

Ludwig, A., Benz, R., Goebel, W.: Oligomerization of Escherichia coli haemolysin (HlyA) is involved in pore formation. Mol. Gen. Genet. 241, 89-96 (1993).

Ludwig, A., Garcia, F., Bauer, S., Jarchau, T., Benz, R., Hoppe, J., Goebel, W.: Analysis of the in vivo activation of hemolysin (HlyA) from Escherichia coli. J. Bacteriol. 178, 5422-5430 (1996).

Ludwig, A., Jarchau, T., Benz, R., Goebel, W.: The repeat domain of Escherichia coli haemolysin (HlyA) is responsible for its $\mathrm{Ca}^{2+}$-dependent binding to erythrocytes. Mol. Gen. Genet. 214, 553-561 (1988).

Ludwig, A., Schmid, A., Benz, R., Goebel, W.: Mutations affecting pore formation by haemolysin from Escherichia coli. Mol. Gen. Genet. 226, 198-208 (1991).

Macdonald, J., Rycroft, A. N.: Molecular cloning and expression of $p t x A$, the gene encoding the 120 - kilodalton cytotoxin of Actinobacillus pleuropneumoniae serotype 2. Infect. Immun. 60, 2726-2732 (1992).

Maheswaran, S. K., Weiss, D. J., Kannan, M. S., Townsend, E. L., Reddy, K. R., Whiteley, L. O., Srikumaran, S.: Effects of Pasteurella haemolytica A1 leukotoxin on bovine neutrophils: degranulation and generation of oxygen-derived free radicals. Vet. Immunol. Immunopathol. 33, 51-68 (1992).

Maier, E., Reinhard, N., Benz, R., Frey, J.: Channelforming activity and channel size of the RTX toxins ApxI, ApxII, and ApxIII of Actinobacillus pleuropneumoniae. Infect. Immun. 64, 4415-4423 (1996).

Osicka, R., Kalmusova, J., Krizova, P., Sebo, P.: Neisseria meningitidis RTX protein FrpC induces high levels of serum antibodies during invasive disease: polymorphism of $\mathrm{rp} C$ alleles and purification of recombinant FrpC. Infect. Immun. 69, 5509 - 5519 (2001).

Reimer, D., Frey, J., Jansen, R., Veit, H. P., Inzana, T. J.: Molecular investigation of the role of ApxI and ApxII in the virulence of Actinobacillus pleuropneumoniae serotype 5. Microb. Pathog. 18, 197-209 (1995).

Rycroft, A. N., Williams, D., Cullen, J. M., Macdonald, J.: The cytotoxin of Actinobacillus pleuropneumoniae (pleurotoxin) is distinct from the haemolysin and is associated with a $120 \mathrm{kDa}$ polypeptide. J. Gen. Microbiol. 137, 561-568 (1991).

Saitou, N., Nei, M.: The neighbor-joining method: a new method for reconstructing phylogenetic trees. Mol. Biol. Evol. 4, 406-425 (1987).

Samitz, E. M., Biberstein, E. L.: Actinobacillus suis-like organisms and evidence of hemolytic strains of Actinobacillus lignieresii in horses. Am. J. Vet. Res. 52, $1245-1251$ (1991).

Schaller, A., Djordjevic, S. P., Eamens, G. J., Forbes, W. A., Kuhn, R., Kuhnert, P., Gottschalk, M., Nicolet, J., Frey, J.: Identification and detection of Actinobacillus pleuropneumoniae by PCR based on the gene apxIVA. Vet. Microbiol. 79, 47-62 (2001).

Schaller, A., Kuhn, R., Kuhnert, P., Nicolet, J., Anderson, T. J., MacInnes, J. I., Segers, R. P. A. M., Frey, J.: Characterization of apxIVA, a new RTX determinant of Actinobacillus pleuropneumoniae. Microbiology 145, $2105-2116$ (1999).

Schaller, A., Kuhnert, P., de la Puente-Redondo, V. A., Nicolet, J., Frey, J.: Apx toxins in Pasteurellaceae species from animals. Vet. Microbiol. 74, 365-376 (2000).

Soloaga, A., Veiga, M. P., Garcia-Segura, L. M., Ostolaza, H., Brasseur, R., Goni, F. M.: Insertion of Escherichia coli alpha-haemolysin in lipid bilayers as a non-transmembrane integral protein: prediction and experiment. Mol. Microbiol. 31, 1013 -1024 (1999).

Stanley, P., Koronakis, V., Hughes, C.: Acylation of Escherichia coli hemolysin: A unique protein lipidation mechanism underlying toxin function. Microbiol. Mol. Biol. Rev. 62, 309-333 (1998).

Stevens, P. K., Czuprynski, C. J.: Pasteurella haemolytica leukotoxin induces bovine leukocytes to undergo morphologic changes consistent with apoptosis in vitro. Infect. Immun. 64, 2687-2694 (1996). 
Strathdee, C. A., Lo, R. Y.: Cloning, nucleotide sequence, and characterization of genes encoding the secretion function of the Pasteurella haemolytica leukotoxin determinant. J. Bacteriol. 171, 916-928 (1989).

Sun, Y., Clinkenbeard, K. D., Clarke, C., Cudd, L., Highlander, S. K., Dabo, S. M.: Pasteurella haemolytica leukotoxin induced apoptosis of bovine lymphocytes involves DNA fragmentation. Vet. Microbiol. 65, 153-166 (1999).

Tatum, F. M., Briggs, R. E., Sreevatsan, S. S., Zehr, E. S., Hsuan, S. L., Whiteley, L. O., Ames, T. R., Maheswaran, S. K.: Construction of an isogenic leukotoxin deletion mutant of Pasteurella haemolytica serotype 1: characterization and virulence. Microb. Pathog. 24, 37-46 (1998).

Thanabalu, T., Koronakis, E., Hughes, C., Koronakis, V.: Substrate-induced assembly of a contiguous channel for protein export from E. coli: reversible bridging of an inner-membrane translocase to an outer membrane exit pore. EMBO J. 17, 6487-6496 (1998).
Udeze, F. A., Kadis, S.: Effects of Actinobacillus pleuropneumoniae hemolysin on porcine neutrophil function. Infect. Immun. 60, 1558 - 1567 (1992).

van den Bosch, J. F., Pennings, A. M. M. A., Cuiijpers, M. E. C. M., Pubben, A. N. B., van Vugt, F. G. A., van der Linden, M. F. I.: Heterologous protection induced by an A. pleuropneumoniae subunit vaccine. Proceedings of the International Pig Veterinary Society Congress 11, 11 (1990).

VanOstaaijen, J., Frey, J., Rosendal, S., MacInnes, J. I.: Actinobacillus suis strains isolated from healthy and diseased swine are clonal and carry apxICABD(var. suis) and apxIICA(var. suis) toxin genes. J. Clin. Microbiol. 35, 1131-1137 (1997).

Welch, R. A.: Pore-forming cytolysins of gram-negative bacteria. Mol. Microbiol. 5, 521 - 528 (1991).

Welch, R. A.: RTX toxin structure and function: a story of numerous anomalies and few analogies in toxin biology. Curr. Top. Microbiol. Immunol. 257, $85-$ 111 (2001). 